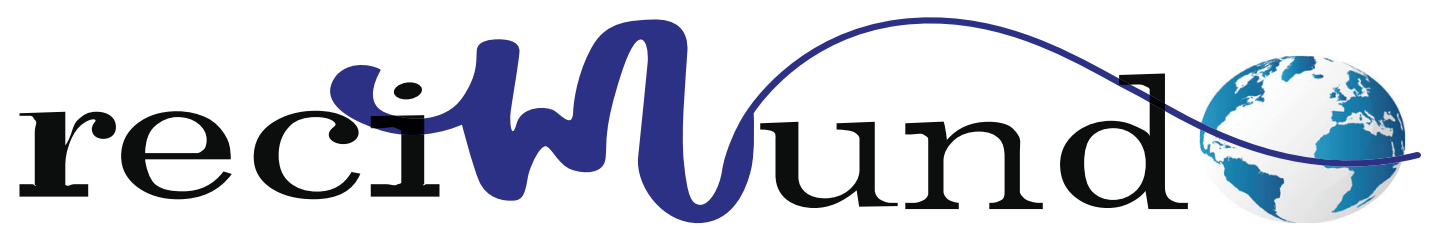

Revista Científica Mundo de la Investigación y el Conocimiento

DOI: 10.26820/recimundo/4.(4).noviembre.2020.156-167

URL: http://recimundo.com/index.php/es/article/view/935

EDITORIAL: Saberes del Conocimiento

REVISTA: RECIMUNDO

ISSN: 2588-073X

TIPO DE INVESTIGACIÓN: Reporte de caso

CÓDIGO UNESCO: 32 Ciencias Médicas

PAGINAS: $156-167$

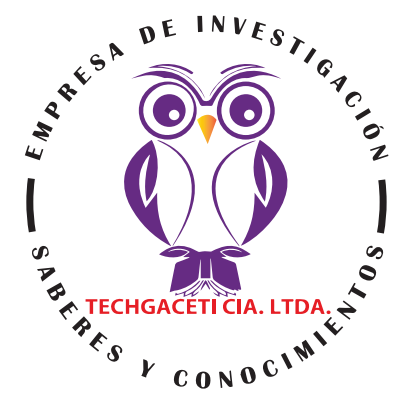

\title{
Manejo híbrido de aneurismas cerebrales gigantes. A propósito de un caso
}

Hybrid management of giant brain aneurysms. About a case

Tratamento híbrido de aneurismas cerebrais gigantes. Sobre um caso

Dr. Kléber Eduardo González Echeverría'; Dr. Héctor Enrique Delgado Zambrano;

Est. Zuly Vanessa Endara Garcíä; Dr. Juan Carlos Calderón León ${ }^{4}$

\section{RECIBIDO: 30/08/2020 ACEPTADO: 02/09/2020 PUBLICADO: 10/11/2020}

1. Especialista en Medicina Neurocirugía; Médico; Investigador Independiente; Guayaquil, Ecuador; Klerdolez@gmail.com; (iD) https://orcid.org/0000-0003-1634-5305

2. Médico Cirujano; Universidad Espíritu Santo; Guayaquil, Ecuador; hectordelgado@uess.edu.ec; (iD https://orcid.org/00000001-6301-9284

3. Médico; Investigadora Independiente; Guayaquil, Ecuador; zulyvanne428@gmail.com; iD https://orcid.org/0000-00015817-6963

4. Médico; Universidad Espíritu Santo; Guayaquil, Ecuador; jcalderonleon@uess.edu.ec; (iD) https://orcid.org/0000-00032663-6069

CORRESPONDENCIA

Kléber Eduardo González Echeverría

klerdolez@gmail.com

Guayaquil, Ecuador 


\section{RESUMEN}

Los aneurismas cerebrales gigantes son aquellos que alcanzan un tamaño mayor de $25 \mathrm{~mm}$ que aparte de poder romperse pueden provocar efecto de masa comportándose similar a un tumor. La compleja anatomía de la arteria carótida interna, segmento paraclinoideo $(\mathrm{ACl})$ dificulta el tratamiento quirúrgico de los aneurismas que surgen de este segmento. La problemática es la toma de decisiones en el manejo de estos aneurismas, buscando siempre la obliteración y exclusión del aneurisma. Se trata de caso clínico de paciente masculino de 63 años de edad con antecedentes quirúrgicos por aneurisma sacular recanalizado a nivel del segmento oftálmico izquierdo de proyección medial (tratado mediante stents y coils) hace 12 años con abandono de tratamiento e ingesta de alcohol muy frecuente. Durante su hospitalización presenta crisis convulsivas, irritabilidad, se le realizan estudios de imágenes donde se observa aneurisma gigante parciamente ocluido, se diagnostica aneurisma gigante de segmento oftálmico izquierdo - proyección medial, trombosado y calcificado. Se estudia y se programa en una primera instancia para embolización de porción aneurismática recanalizada y en una segunda instancia para procedimiento quirúrgico mediante craneotomía. Se reseca pared externa parcialmente en región anterior, posterior y craneal, porción caudal no se resecó, se continúa con resección de porción calcificada y capas trombóticas que se encuentran en región intramural. Se observa porción aneurismática previamente embolizada, realizando así descompresión a nivel frontal izquierdo. El manejo híbrido de los aneurismas cerebrales gigantes se logra con la combinación de procedimientos endovasculares y cirugía abierta obteniendo como resultado una mejoría clínica del paciente. Se hizo evaluaciones cognitivas y sus capacidades se demostraron en cualificación positivas. Desaparecieron sus cuadros de conducta: irritabilidad, apatía y mal manejo de la frustración. Al momento se encuentra estable en sus emociones, en la recuperación psicológica-afectiva-cognitiva adecuada.

Palabras clave: aneurisma cerebral gigante, craneotomía, procedimiento endovascular.

\section{ABSTRACT}

Giant brain aneurysms are those that reach a size greater than $25 \mathrm{~mm}$ that apart from being able to rupture can cause a mass effect behaving similar to a tumor. The complex anatomy of the paraclinoid internal carotid artery (ICA) makes surgical treatment of aneurysms arising from this segment difficult. The problem is the decision-making in the management of these aneurysms, always seeking the obliteration and exclusion of the aneurysm. This is a clinical case of a 63-year-old male patient with a surgical history for recanalized saccular aneurysm at the level of the medial projection ophthalmic segment (treated with stents and coils) 12 years ago, with very frequent treatment and alcohol abandonment. During his hospitalization, he presents seizures, irritability, imaging studies are performed where a partially clipped giant aneurysm is observed, a giant aneurysm of the left ophthalmic segment is diagnosed - medial projection, thrombosed and calcified. It is studied and scheduled for surgical procedure. Partial capsule resection, calcified portion and thrombotic layers are resected. A previously embolized aneurysmal portion is observed. Hybrid management of giant cerebral aneurysms is achieved with the combination of endovascular procedures and surgery, resulting in clinical improvement of the patient. Cognitive evaluations were made and their abilities were demonstrated in positive qualification. His patterns of behavior disappeared: irritability, apathy and mismanagement of frustration. At the moment he is stable in his emotions, in the adequate psychological-affective-cognitive recovery.

Keywords: globular intracranial aneurysm, clinical case, surgery, endovascular procedure.

\section{RESUMO}

Aneurismas cerebrais gigantes são aqueles que atingem um tamanho maior que $25 \mathrm{~mm}$ que além de poderem se romper podem causar um efeito de massa com comportamento semelhante a um tumor. A complexa anatomia da artéria carótida interna paraclinóide $(\mathrm{ACl})$ dificulta o tratamento cirúrgico dos aneurismas decorrentes deste segmento. O problema é a tomada de decisão no manejo desses aneurismas, sempre visando a obliteração e exclusão do aneurisma. Trata-se de um caso clínico de um paciente do sexo masculino, 63 anos, com história cirúrgica de aneurisma sacular recanalizado ao nível do segmento oftálmico de projeção medial (tratado com stents e molas) há 12 anos, com tratamento muito frequente e abandono do álcool. Durante a internação apresenta convulsões, irritabilidade, são realizados exames de imagem onde se observa aneurisma gigante parcialmente clipado, é diagnosticado aneurisma gigante do segmento oftálmico esquerdo - projeção medial, trombosado e calcificado. É estudado e programado para procedimento cirúrgico. Ressecção parcial da cápsula, porção calcificada e camadas trombóticas são ressecadas. Observa-se uma porção aneurismática previamente embolizada. O manejo híbrido dos aneurismas cerebrais gigantes é alcançado com a combinação de procedimentos endovasculares e cirurgia, resultando na melhora clínica do paciente. Avaliações cognitivas foram feitas e suas habilidades foram demonstradas em qualificação positiva. Seus padrões de comportamento desapareceram: irritabilidade, apatia e má gestão da frustração. No momento ele está estável em suas emoções, na adequada recuperação psicológico-afetivo-cognitiva.

Palavras-chave: aneurisma intracraniano globular, caso clínico, cirurgia, procedimento endovascular. 
J. C.

\section{Introducción}

Los aneurismas cerebrales gigantes son aquellos que alcanzan un tamaño mayor de $25 \mathrm{~mm}$ que aparte de poder romperse pueden provocar efecto de masa comportándose similar a un tumor. Hay diferentes tipos de aneurismas, pero la gran mayoría son por causas hereditarias, por tabaquismo, alcohol y consumo de drogas. Esto genera una serie de síntomas que van desde cefalea, perdida del conocimiento hasta comunicación incoherente. Los tratamientos son diversos y depende de las características del aneurisma y del paciente (Valverde Zambrano, Mora Muñoz, Calderón Moreira, \& Sarmiento, 2019).

Los aneurismas gigantes representan aproximadamente del 5 al $8 \%$ de todos los aneurismas intracraneales y su distribución aproximada es la siguiente: $60 \%$ en la arteria carótida interna, $10 \%$ en la arteria comunicante anterior, $10 \%$ en la arteria cerebral media, $15 \%$ en la arteria basilar y $5 \%$ en las arterias vertebrales. La sintomatología no solo depende del sangrado, el cual ocurre en el $25 \%$ de los casos, sino del efecto de masa del aneurisma, observándose cefaleas, convulsiones, trastornos motores, alteraciones campimétricas y disfunción de nervios craneales hasta en el $70 \%$ de los casos.

La compleja anatomía de la arteria carótida interna paraclinoidea $(\mathrm{ACl})$ dificulta el tratamiento quirúrgico de los aneurismas que surgen de este segmento. El manejo endovascular se ha convertido en la medida terapéutica de primera opción (Roja, y otros, 2019).

La problemática es la toma de decisiones en el manejo de estos aneurismas, buscando siempre la obliteración y exclusión del aneurisma. Existen varias opciones de tratamiento, incluyendo quirúrgicas, endovasculares y la combinación de ambas.

Los procedimientos quirúrgicos son los más aceptados, e incluyen: clipaje del cuello del aneurisma con o sin oclusión temporal del vaso principal, recubrimiento con acrílico o músculo, ligadura proximal y distal del vaso principal con puentes vasculares extracraneales y colocación de puentes vasculares intracraneales (bypass cerebral).

La elección de la opción quirúrgica depende del grosor y dureza de la pared, del tamaño del cuello y de la importancia de las arterias que estén anatómicamente involucradas. Las técnicas endovasculares se reservan para aquellos pacientes que no puedan ser sometidos a cirugía por factores generales (edad, riesgo cardiovascular) o porque la localización del aneurisma implica alto riesgo al acceso quirúrgico.

\section{Caso clíninco}

Paciente masculino de 63 años de edad.

- App: HTA, diabetes mellitus, aneurisma cerebral.

- Antecedentes quirúrgicos: aneurisma sacular recanalizado a nivel del segmento oftálmico izquierdo de proyección medial (tratado mediante stents y coils) hace 12 años.

- Alergias: no refiere.

- Medicación habitual: abandono de tratamiento.

- Hábitos: alcohol muy frecuente.

- Motivo de consulta: Disnea, sincope, convulsiones.

\section{Enfermedad actual}

Masculino de 63 años de edad que ingresa por cuadro clínico de 2 horas de evolución caracterizado por sincope con recuperación de conciencia en 10 seg. Sin relajación de esfínteres, además de disnea en reposo clase funcional IV/IV, taquipnea fr: $26 \times \mathrm{min}$, además al momento refiere dolor precordial leve de intensidad 2/10 de tipo opresivo que no se irradia, presenta edema de miembros inferiores, hemodinamia normotenso mecánica ventilatoria regular, por lo que acude 
a casa de salud donde le refieren a clínica San Francisco de la ciudad de Guayaquil. Durante su hospitalización presenta convulsiones, irritabilidad, le realizan estudios de imágenes donde se observa aneurisma gigante recanalizado, se estudia y se programa para procedimiento quirúrgico.

Exámenes complementarios:

\section{Ecocardiograma}

Disfunción sistólica ventricular izquierda leve (40\%).

Cardiopatía isquémica, disfunción diastó- lica restrictiva tipo III, dilatación moderada de Al, valvulopatía mitro-aórtica moderada, valvulopatía tricuspídea leve, HTP moderada.

\section{Angiotomografía cerebral}

Aneurisma gigante, con calcificaciones centrales y periféricas, de localización frontal izquierda, que mide $5 \times 6 \times 6 \mathrm{~cm}(T \times \mathrm{CC}$ $x$ AP). Artefactos metálicos por material de embolización en su raíz.

Imagen 1 y 2. TAC de cerebro simple - contrastada. Aneurisma gigante, con calcificaciones centrales y periféricas.

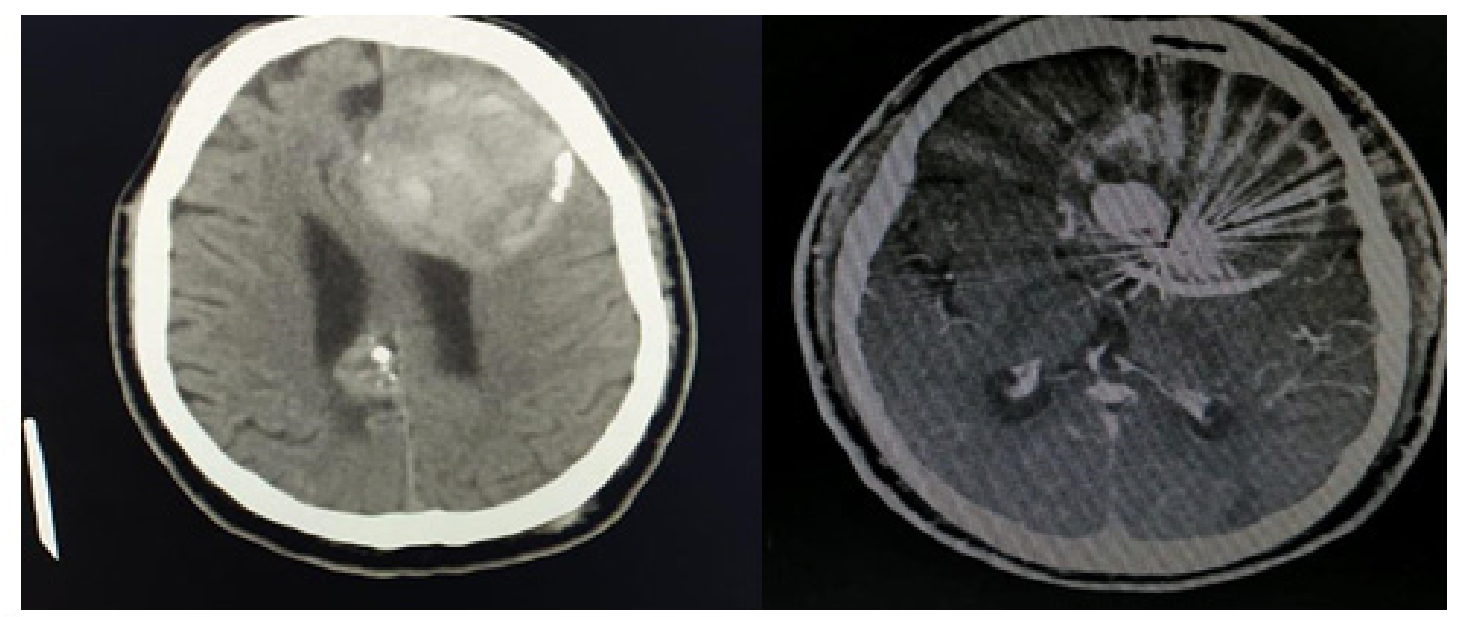

Fuente: Los autores

Luego de la administración de contraste IV se evidencia paso de contraste hacia aneurisma, con un volumen aproximado de $5 \mathrm{cc}$. El aneurisma desplaza y comprime el lóbulo frontal izquierdo y determina herniación subfalcina. Desviación de la línea media hacia la derecha: $16 \mathrm{~mm}$. 
GONZÁLEZ ECHEVERRÍA, K. E., DELGADO ZAMBRANO, H. E., ENDARA GARCÍA, Z. V., \& CALDERÓN LEÓN, J. C.

Imagen 3. Aneurisma gigante de localización frontal izquierda.

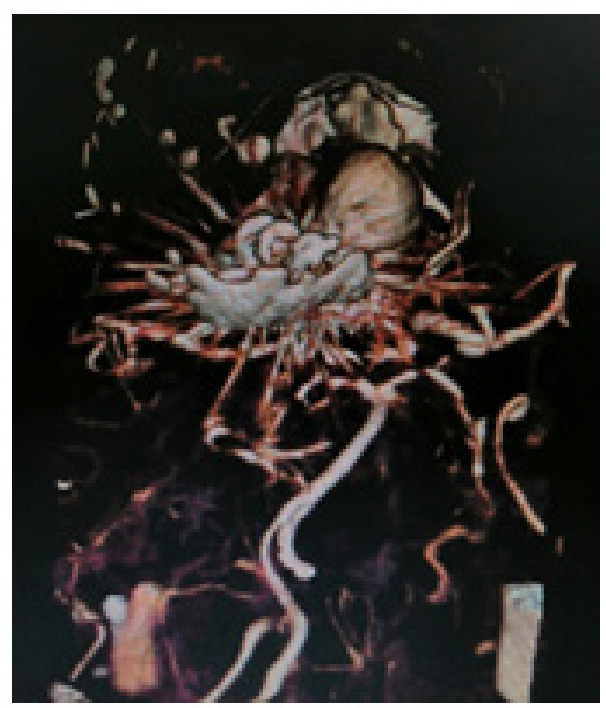

Fuente: Los autores

Imagen 4. Aneurisma gigante de localización frontal izquierda, que mide $5 \times 6$ ×6cm (T $x$ CC x AP).
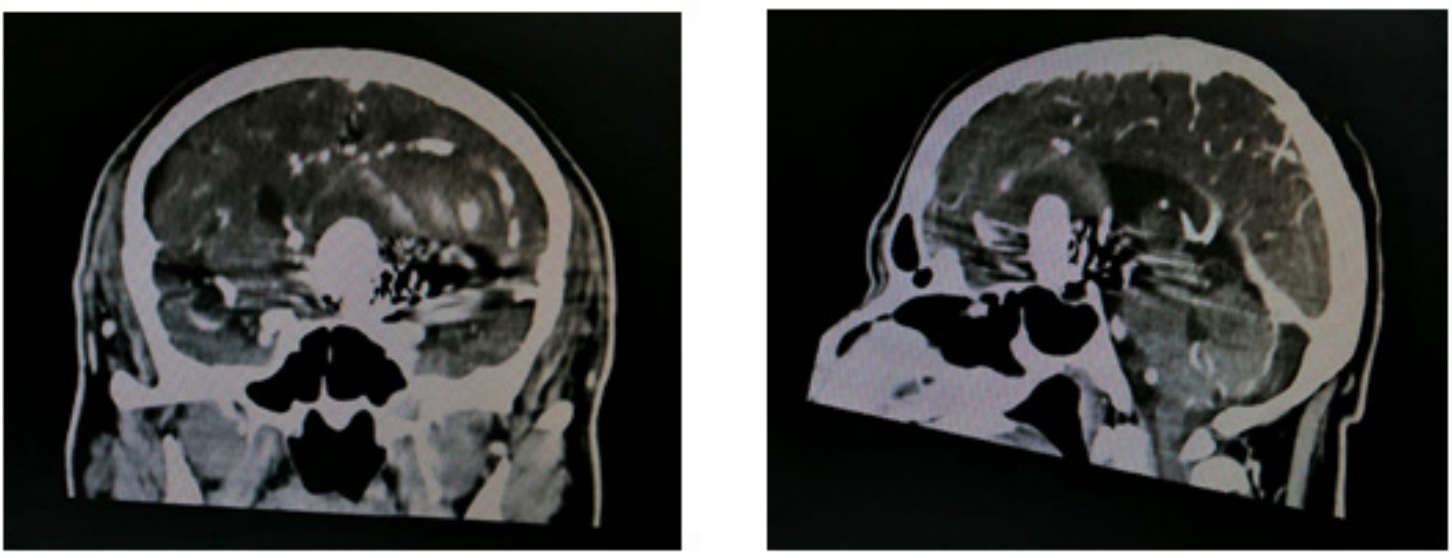

Fuente: Los autores

Aneurisma gigante, con calcificaciones $x$ AP). Artefactos metálicos por material de centrales y periféricas, de localización fronembolización en su raíz.

tal izquierda, que mide $5 \times 6 \times 6 \mathrm{~cm}(\mathrm{~T} \times \mathrm{CC}$ 
Imagen 5. Artefactos metálicos por material de embolización en su raíz.

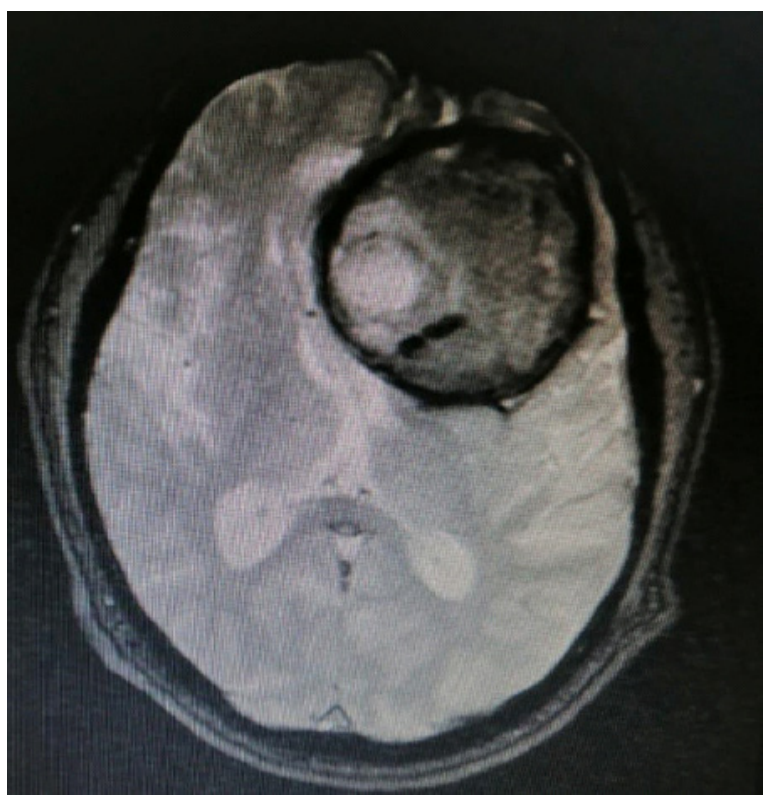

Fuente: Los autores

Imagen 6. Flujo central en aneurisma, con un volumen aproximado de 5cc

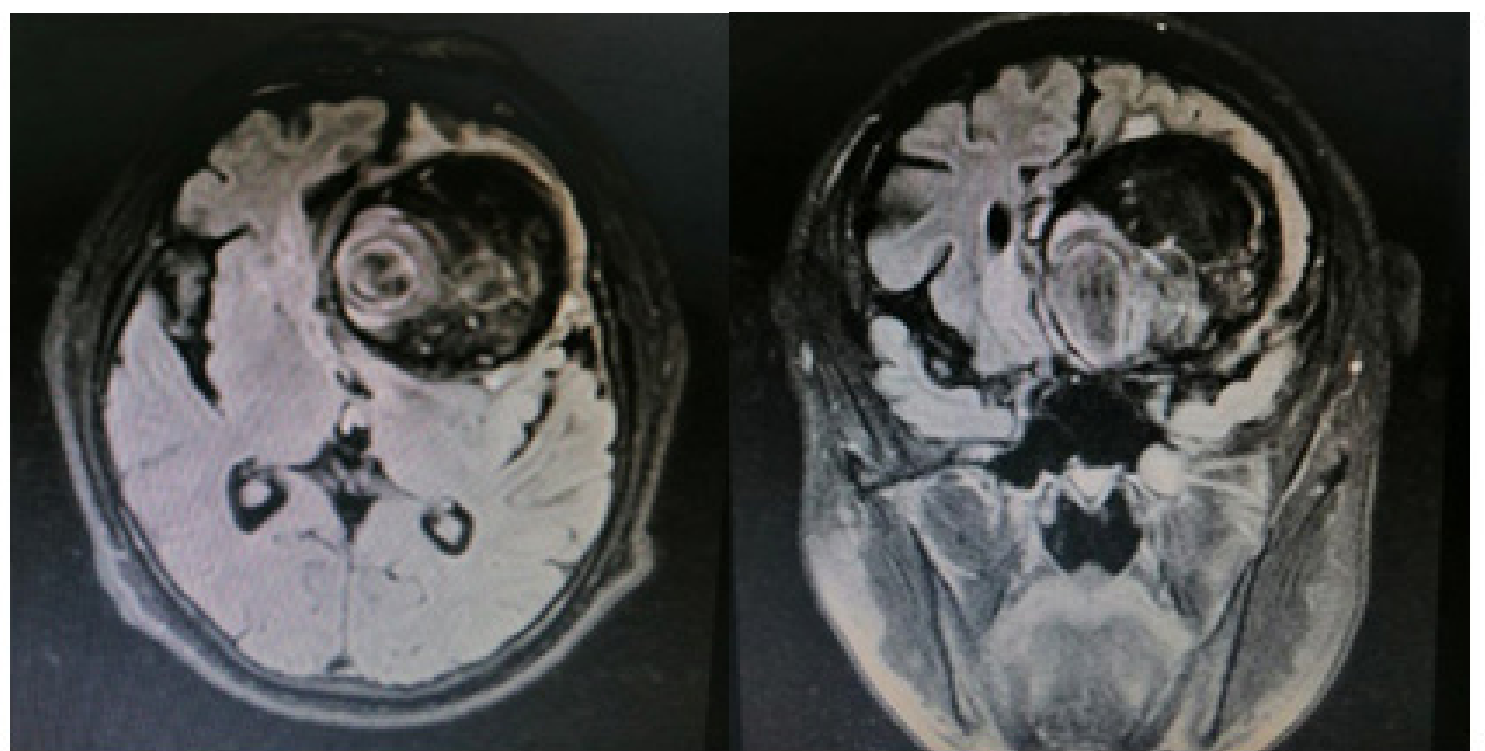

Fuente: Los autores

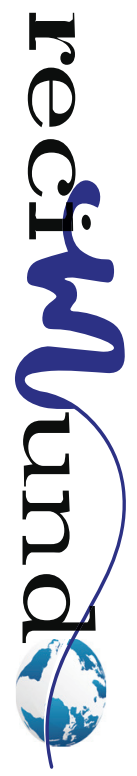




\section{J. C.}

Luego de la administración de contraste IV se evidencia paso de contraste hacia aneurisma, con un volumen aproximado de 5cc. El aneurisma desplaza y comprime el lóbulo frontal izquierdo y determina herniación subfalcina. Desviación de la línea media hacia la derecha: $16 \mathrm{~mm}$. Realce de las estructuras arteriales a nivel del polígono de Willis así como del sistema vertebro basilar, carótidas intra y extracraneanas. Artefactos por pulso adyacentes. El aneurisma desplaza y comprime el lóbulo frontal izquierdo y determina herniación subfalcina. Desviación de la línea media hacia la derecha: $16 \mathrm{~mm}$.

Procedimiento Endovascular, Colocación de14 Coils.

Imagen 7. Recanalización de Aneurisma y flujo adyacente
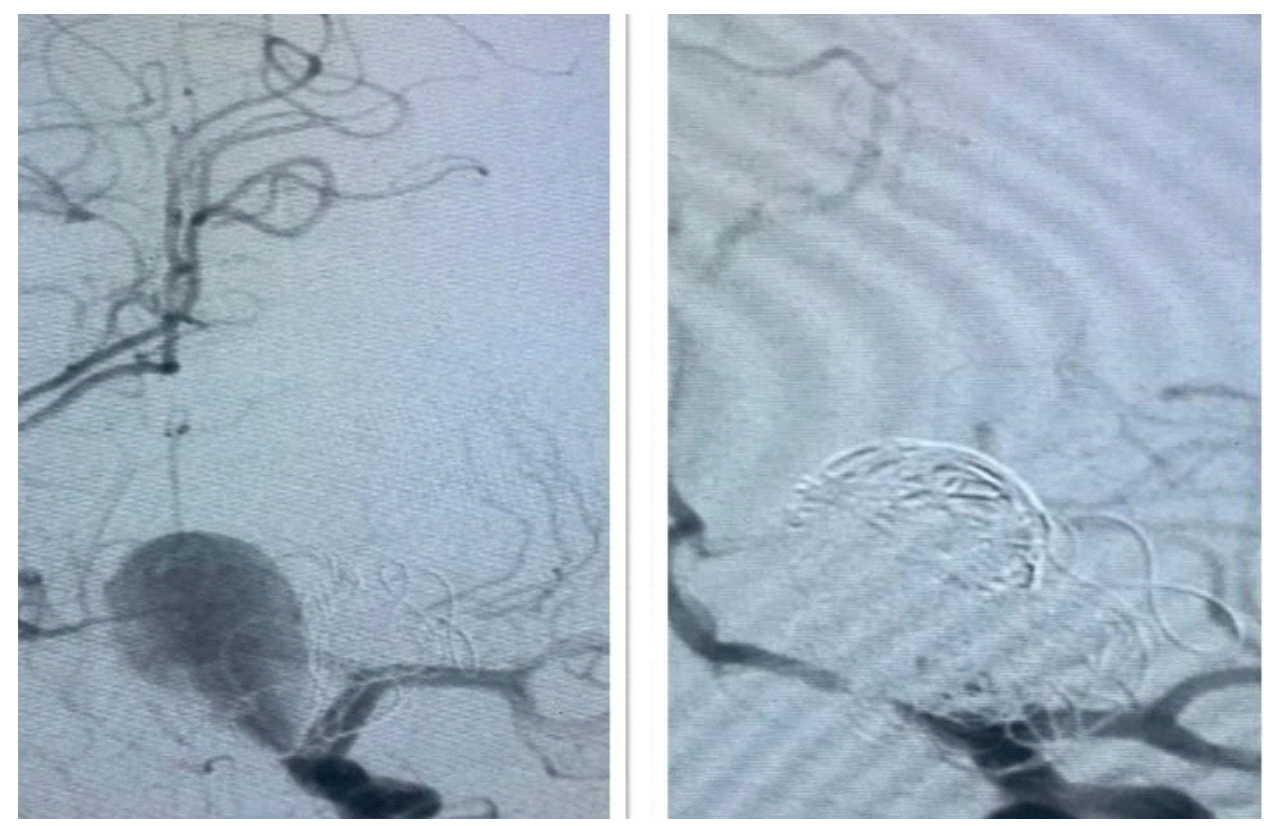

Fuente: Los autores

Aneurisma embolizado arteria carótida interna izquierda, aneurisma recanalizado a nivel del segmento oftálmico izquierdo, dicha recanalización posee las siguientes medidas: longitud $40 \mathrm{~mm}$, ancho $30 \mathrm{~mm}$ y cuello $5 \mathrm{~mm}$. No hay evidencia de vasoespasmo.
CONCLUSIÓN: aneurisma cerebral recanalizado a nivel del segmento oftálmico izquierdo donde se colocaron 14 Coils con lo que se logra la oclusión completa de la lesión aneurismática. 
Imagen 8. Resultado de patología.

\title{
EXAMEN MACROSCOPICO
}

\begin{abstract}
SE RECIBEN VARIOS FRAGMENTOS DE TZJIDO DE CONSISTENCLA BLANDA COLOR AMARIIIENTO ROSADO, QIE EN CONTINTO MIDE 2.3 CM, SE PROCESA INTEGRAWIENTE PARA SI ESTLDIO IIISTOPATOLOGICO.
\end{abstract}

\section{EXAMEN MICROSCOPICO}

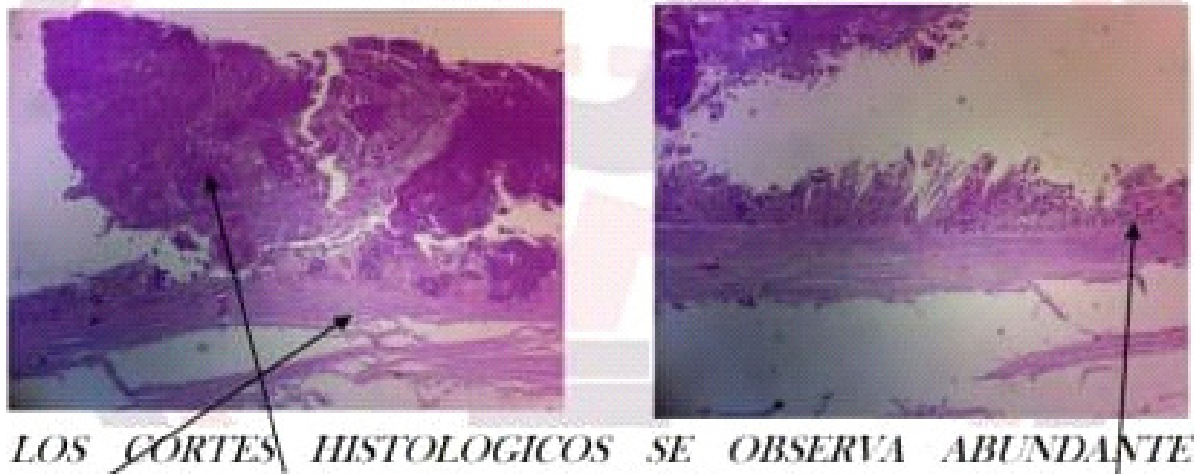
MSTERLAL HEMATTCO, TEIIDO GLLAL, Y ESTRECTTRA VASCULAR CON ADELGAZAMIENTO DE LA MISCULARIS PROPLA, CON FOCOS MIIROSCOPICOS DE CALFICICACION. EN LOS CORTES ESTTDLADOS NO SE IDENTIFICAN CAMBIOS NEOPLASICOS MAIIGNOS.

DIAGNOSTICO

- HALIAZGOS HISTOLOGICOS CONGRUENTES CON LESION ANEURISMATICA CON FOCOS DE CALCIFICACION.

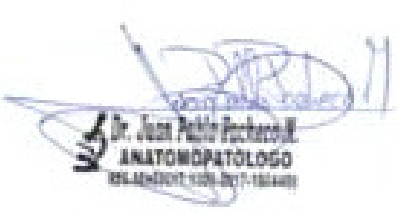

Fuente: Los autores

\section{PROCEDIMIENTOS QUIRÚRGICOS:}

\section{Reporte de arteriografía cerebral}

Arteria carótida común derecha, previo roadmapping se cateteriza la a. Carótida interna y se realizan proyecciones angiográficas convencionales que revelan vasculatura intracraneal sin evidencia de lesión angiográfica. Como hallazgo se observa desplazamiento de la A. Cerebral anterior derecha en sentido lateromedial (de izquierda a derecha $>$ de $20 \mathrm{~mm}$ ).

\section{Reporte de Embolización}

Una vez realizado el diagnóstico, se coloca catéter guía a nivel de la A. Carótida inter- na izquierda, perfundimos catéter guía con solución salina a razón de 400 ml hora mediante utilización de llave en "y", se realiza roadmapping de carótida interna y se sube microcatéter (echelon 10) montado en microguía (runthrough) con lo que se llega al saco aneurismático (atravesando los stent carotideos) y luego de colocar 14 coils (microlux 15-20 mm x $60 \mathrm{~cm}$, microlux 15-20 $\mathrm{mm} \times 40 \mathrm{~cm}$ y microlux 6-10 $\mathrm{mm} \times 30 \mathrm{~cm}$ con su respectivo liberador $\mathrm{V}$-Gripa. Penumbra Smart coila $14 \mathrm{~mm} \times 60 \mathrm{~cm}$, penumbra Smart coila $11 \mathrm{~mm}$ x $60 \mathrm{~cm}$ con su liberador, detachment handle. Jasper coila 3D $9 \mathrm{~mm} \times$ $40 \mathrm{~cm}$, jasper coila $8 \mathrm{~mm} \times 30 \mathrm{~cm}$, jasper 6 $\mathrm{mm} \times 20 \mathrm{~cm}$, jasper $5 \mathrm{~mm} \times 20 \mathrm{~cm}$, con su respectivo set de cables liberadores. Optima coila sistem $8 \mathrm{~mm} \times 30 \mathrm{~cm}$, optima cois

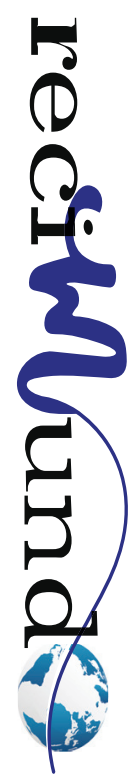



J.C.

sistem $7 \mathrm{~mm} \times 30 \mathrm{~cm}$, optima cois sistem 6 $\mathrm{mm} \times 20 \mathrm{~cm}$ con su liberador xcel. Axium coila $14 \mathrm{~mm} \times 30 \mathrm{~cm}$ y axium cois $12 \mathrm{~mm} \times$ $40 \mathrm{~cm}$, con sus liberador instant detacher) con lo que se logra la oclusión completa de la lesión aneurismática.

\section{Craneotomía}

Aneurisma gigante de segmento oftálmico izquierdo - proyección medial, trombosado y calcificado. Se reseca cápsula parcialmente, resección de porción calcificada y capas trombóticas. Se observa porción aneurismática previamente embolizada.

Paciente que ingresa desde el área de terapia intensiva: bajo efectos de anestesia

Imagen 9. Abordaje pterional izquierdo.

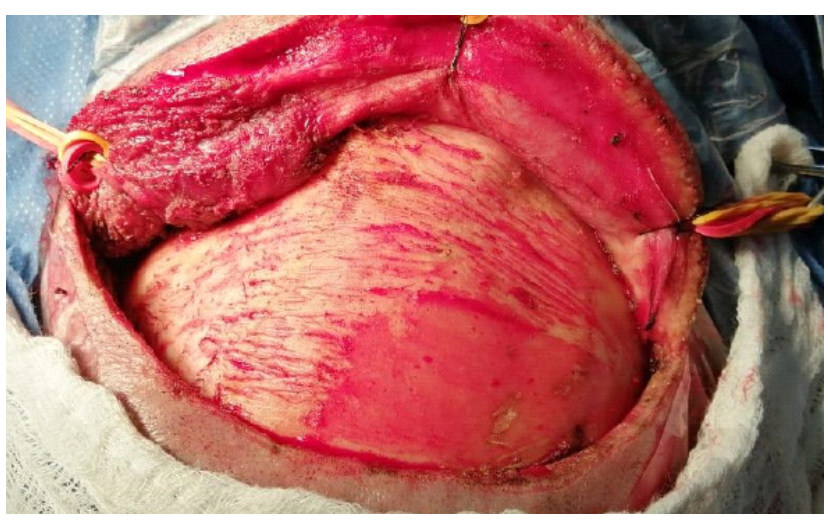

Fuente: Los autores

Imagen 11. Apertura dural.

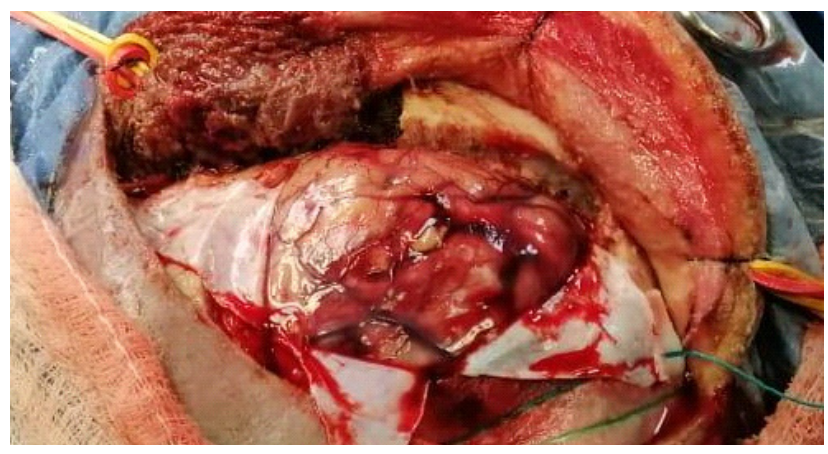

Fuente: Los autores general se coloca al paciente en decúbito dorsal con cefaloversión a la derecha, se realiza asepsia y antisepsia.

Colocación de campos quirúrgicos estériles, se realiza incisión pterional izquierda, craneotomía frontal izquierda, apertura dural en forma de estrella, se realiza corticotomia frontal izquierda observándose aneurisma trombosado y calcificado, se reseca cápsula parcialmente, resección de porción calcificada y capas trombóticas. Se observa porción aneurismática previamente embolizada. Finalmente se realiza craneoplastia con duragen de $7.5 \times 5.5 \mathrm{cms}$., se coloca plaqueta ósea sobre superficie dural.

Imagen 10. Retiro de Plaqueta Ósea

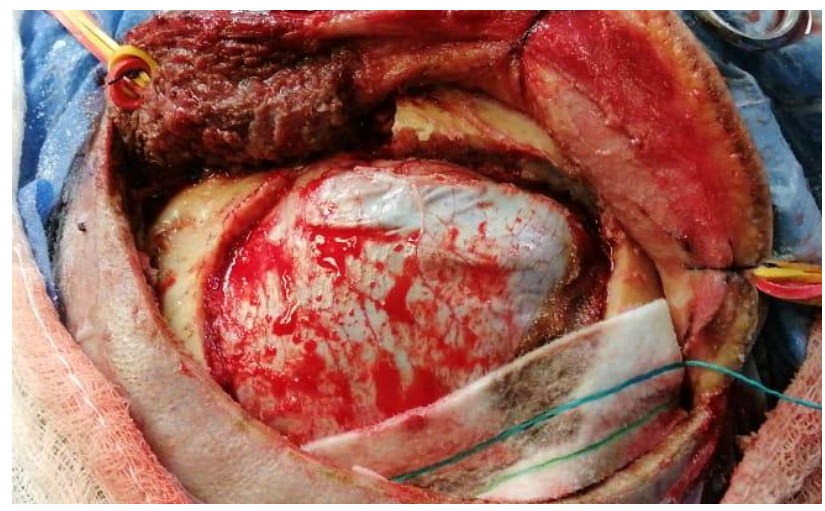

Fuente: Los autores

Imagen 12. Corticotomía para abordaje a aneurisma gigante. Obsérvese el gran edema que existe del parénquima cerebral adyacente.

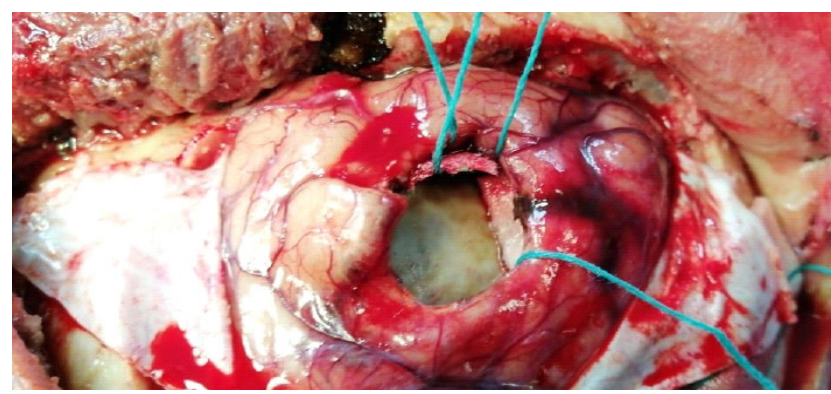

Fuente: Los autores 
Se utiliza micro disección con disectores de trombos intramurales. rothon. Se retiran porciones calcificadas y

Imagen 13. Retiro de pared externa de aneurisma y aspiración de trombo intramural:

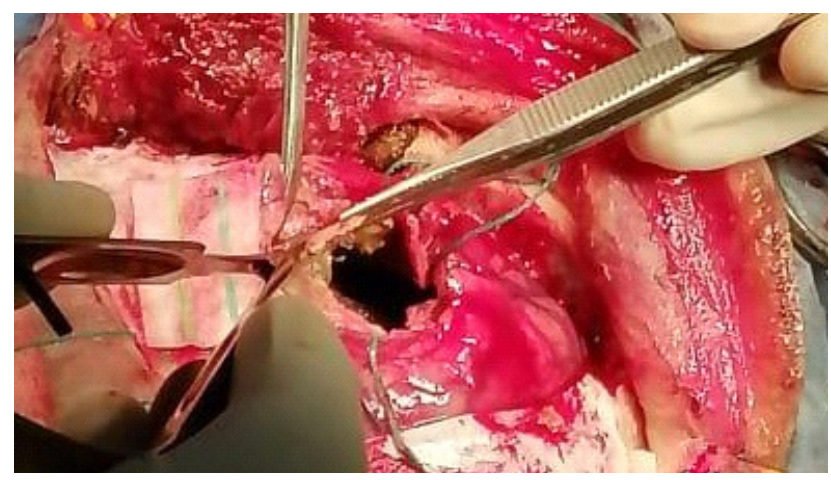

Fuente: Los autores

Se reseca pared externa parcialmente hasta observar porción aneurismática previamente embolizada. Se lava con abundante solución salina y se realiza hemostasia con surgicel.

Imagen 14. Aspiración de trombo intramural.



Fuente: Los autores

Imagen 15. Colocación de plaqueta ósea fijado con cráneo cover.



Fuente: Los autores 
J.C.

Se cierra por planos con vicryl 1.0 hasta subcutáneo, se cierra piel con nylon 3/0 cubriendo con apósito estéril. Se termina cirugía tiempo transquirúrgico 3 horas.

\section{Conclusiones}

\section{Evolución del paciente}

Previo a los procedimientos quirúrgicos, el paciente presentaba convulsiones, irritabilidad, dificultad respiratoria y ceguera total izquierda. Se realiza en primera instancia pan arteriografía con colocación de 14 Coils y luego se programa para craneotomía con resección de porción calcificada y trombótica de la pared del aneurisma la cual por su complejidad se prepara al paciente previo consentimiento familiar, se explican riesgos y beneficios los cuales acceden al tratamiento quirúrgico, se coloca vía central, línea arterial para su monitorización, ingresa a quirófano con anestesia general, transquirúrgico de tres horas, sangrado escaso sin complicaciones, pasa al área de terapia intensiva despierto, se mantiene estable por lo que después de 72 horas pasa a piso con evolución favorable, hemodinámica conservada y luego dado de alta.

Posterior a tratamiento quirúrgico paciente mejoró su estado general, trastorno conductuales, menos irritable, no ha convulsionado, persiste ceguera total izquierda, la cual si se hubiera tratado adecuadamente hace 12 años no se hubiera presentado y esto secundario al efecto de masa que causaba dicho aneurisma.

\section{Evaluación neuropsicológica}

El paciente en el antecedente psicológico, demostraba tener irritabilidad, cambios repentinos de humor, demostraba sensibilidad a la frustración, según lo manifiesta su familia. Ha sido un proceso muy difícil para el paciente. Su familia le ha brindado su apoyo en todo momento, algo que lo ha sentido y permitido sobrellavar con optimismo su situación médica. Se le ha brindado, por parte del terapeuta, el apoyo emocional, manejo de existencialismo, control de su realidad, el manejo lo mejor posible para hacer frente a su situación. Que su familia también mejoró en enfrentar, al aliviar la ansiedad entendiendo adecuadamente los procesos.

En el proceso de evaluación psicológica post cirugía, se puede decir, que sus funciones ejecutivas superiores encuentran mayormente conservadas. Se hizo evaluaciones cognitivas y sus capacidades se demostraron en cualificación positivas. Desaparecieron sus cuadros de conducta: irritabilidad, apatía y mal manejo de la frustración. Dice tener una nueva oportunidad para disfrutar de su familia. El paciente demuestra tener, buena actitud, motivado, dice estar feliz porque su familia estuvo en todo momento apoyándolo, agradecido con el valor humano que la medicina y los doctores le han brindado. Al momento se encuentra estable en sus emociones en compañía de su familia, en la recuperación psicológica-afectiva-cognitiva adecuada.

Concluyendo que el manejo híbrido de los aneurismas cerebrales gigantes se logra en combinación de procedimientos endovascular y la micro neurocirugía, teniendo como resultado gran mejoría clínica del paciente. 


\section{Bibliografía}

Roja, G., rodríguez, R., Durand, W., Vallejos, R., Valger, D., Flores, J., y otros. (2019). Tratamiento endovascular con coil penumbra de aneurisma grande del segmento oftálmico derecho. Peru $\mathrm{J}$ Neurosurg, 14(1), 21-24.

Valverde Zambrano, M. J., Mora Muñoz, M. M., Calderón Moreira, M. C., \& Sarmiento, M. J. (2019). Aneurisma Cerebral. Intervención Quirúrgica de
Microstent, Indicaciones y Resultados. RECIMUNDO: Revista Científica de la Investigación y el Conocimiento, 3(1), 259-286.

Gonzalez NR, Duckwiler G, et al. Challenges in the endovascular treatment of giant intracranial aneurysms. Neurosurgery. 2006;59(S3):113-124. (pubmed).

Spetzler RF, Riina HA, Lemole GM., Jr Giant aneurysms. Neurosurgery. 2001;49:902-908. (pubmed).

\section{CITAR ESTE ARTICULO:}

González Echeverría, K. E., Delgado Zambrano, H. E., Endara García, Z. V., \& Calderón León, J. C. (2020). Manejo híbrido de aneurismas cerebrales gigantes. A propósito de un caso. RECIMUNDO, 4(4), 156-167. https://doi. org/10.26820/recimundo/4.(4).noviembre.2020.156-167 